\title{
Design and Implementation of Intelligent Comprehensive Information Platform for the Aged
}

\author{
Yujia Yang ${ }^{1, a}$, Jie Dong ${ }^{1, b^{*}}$ \\ ${ }^{1}$ Shenyang Jianzhu University. No.9, Hunnan East Road, Hunnan New District, Shenyang City, \\ Liaoning, P.R.China \\ a 879385384@qq.com, b23236930@qq.com
}

Keywords: Elderly Service Information Platform; Intelligent; MVC Architecture

\begin{abstract}
With the increasingly serious form of social aging, the problem of providing for the aged is imminent. The rapid development of internet commerce provides space for the development of the elderly service industry to integrate and upgrade. according to the main problems of the current old-age care website, this paper uses data recommendation technology to designs an intelligent comprehensive information platform for the old-age care, providing old-age care information, health care guidance, old-age goods, as well as various types of old-age care organization information and considerate service information for the old. To a certain extent, it will integrate endowment resources, enrich the endowment system, and realize the convenience of endowment.
\end{abstract}

\section{Introduction}

According to the report, China's aging population will reach 167 million by 2020, accounting for $24 \%$ of the world's aging population. This means that for every four elderly people in the world, one of them is Chinese. The problem of aging population is getting more and more serious, and the problem of providing for the aged needs to be solved urgently. With the rapid development of the internet, it is more convenient to integrate life with the internet. it is very important to design a comprehensive information website for the aged to upgrade the old-age service and solve the oldage problem. The application of web technology to develop a comprehensive information platform for the elderly to provide the elderly with old-age information, health care guidance, commodity purchase, as well as various types of old-age organization information and considerate services. Young people can order corresponding caring services for the elderly or inquire information about nursing homes through the platform, and the elderly can browse and view the corresponding contents themselves. Collect information on endowment resources to provide great convenience for healthy endowment.

\section{The Main Problems of the Existing Pension Platform}

At the beginning of the website design, we also made an investigation on the relevant platforms in the elderly service market, objectively analyzed the advantages and disadvantages of the existing platforms from the perspectives of user demand and user experience, and generally found the following problems that need to be improved.

Insufficient types: the existing elderly service platform is mainly concentrated on information websites such as nursing home publicity website, old-age news website, old-age health care website, etc. There is an extreme lack of information integration of endowment resources.

Insufficient function: the information website for the elderly can only provide information help for the elderly. for the elderly, its function is greatly insufficient. A network-determinable community service for the elderly is unable to provide. Moreover, it lacks detailed suggestions on the health of the elderly and lacks websites that provide considerate services for the elderly.

Lack of personalization: at present, there are few personalized recommendations for news, activities, commodities and so on that the elderly care about.[1] 


\section{System Design}

Overall Structural Design. This system uses MVC design pattern to build enterprise web application. HTML pages, JSP pages, swing GUI correspond to view, because the entire application system mainly interacts with the outside world through views;The controller is servlet class and JavaBean class, which are used to manage the interaction between users and views. JavaBean corresponds to model and provides application business logic to process business data. [2] The web application framework constructed by MVC design pattern is shown in Fig. 1.

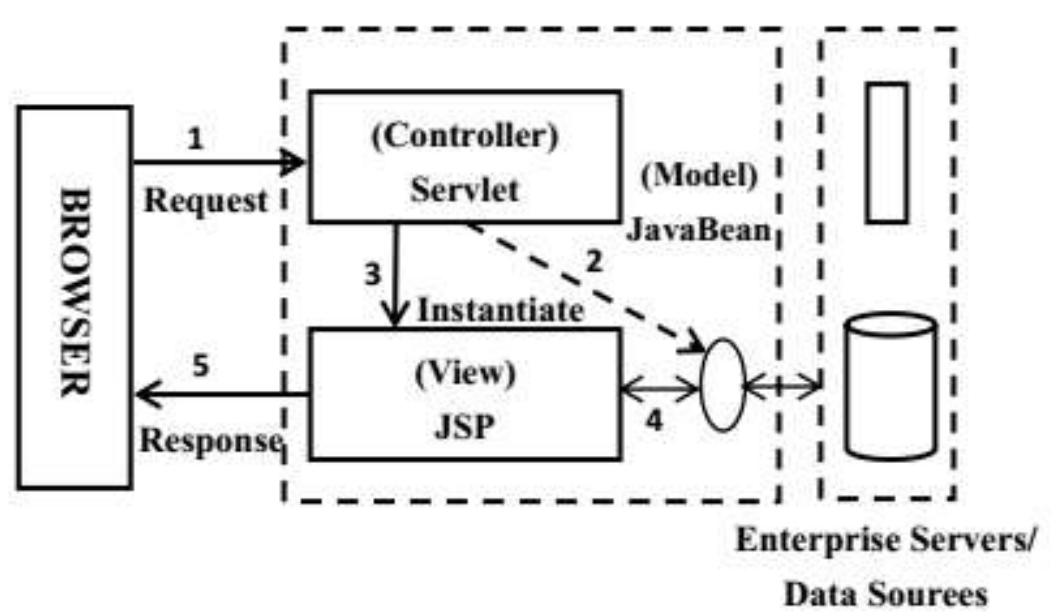

Figure 1. Web application architecture based on Java technology

The MVC system model clearly separates the display of data from the processing of data services, thus making the logical structure clearer. If the display mode of the data changes, only the js view page needs to be changed, and the data processing module does not need to be changed; On the other hand, if the business requirements change, only the corresponding processing data module needs to be changed.

Therefore, the system can easily add new services and can flexibly adapt to changes in various requirements. The MVC design pattern is applied to web applications. The whole process is as follows: when HTML or JSP pages of the web client submit to the server, the server-side controller servlet uniformly processes these submission requests. This controller servlet passes the request to the corresponding service JavaBean operation processing according to the submitted service, and then passes the processing result of the service JavaBean to the view JSP. The view JSP returns to the client in HTML after being processed on the server[3].

System Function Design. The intelligent comprehensive information platform for the aged integrates the information of various old-age institutions, old-age commodities and old-age activities, and provides health management services and health consultation services. Through analyzing the browsing process of users in the information platform for the aged and the commodities and services purchased, the platform understands the needs of the elderly and provides online recommendation functions for the elderly.

Since the system is oriented to system administrators, elderly users and organization, the system is divided into three main functional modules, namely, administrator background module, organization account management module and foreground display module.

The management background module is a functional module designed for administrators to manage the system. Administrators need to $\log$ in to the system before they can manage the system information. Therefore, there must be a login module first. The administrator needs to add, delete and modify the information of the tables in the database after logging into the system. Therefore, a small module is designed for each table under this main function module. The administrator can manage the database information through these modules. 
The organization account management module is a functional module designed for the organization account number to carry out nursing homes and various services under its umbrella. after logging in, the organization account number can register for nursing homes and maintain the rooms under its umbrella. At the same time, it can manage users' nursing home orders and purchase service orders. In addition, it can also manage the setting up of a city for nursing homes and their pictures, etc.

Six sub-modules are designed under the front desk display module: endowment dynamics, endowment institutions, endowment knowledge, endowment commodities, endowment forums, and health index. The functional modules of the website are shown in Fig. 2 below.

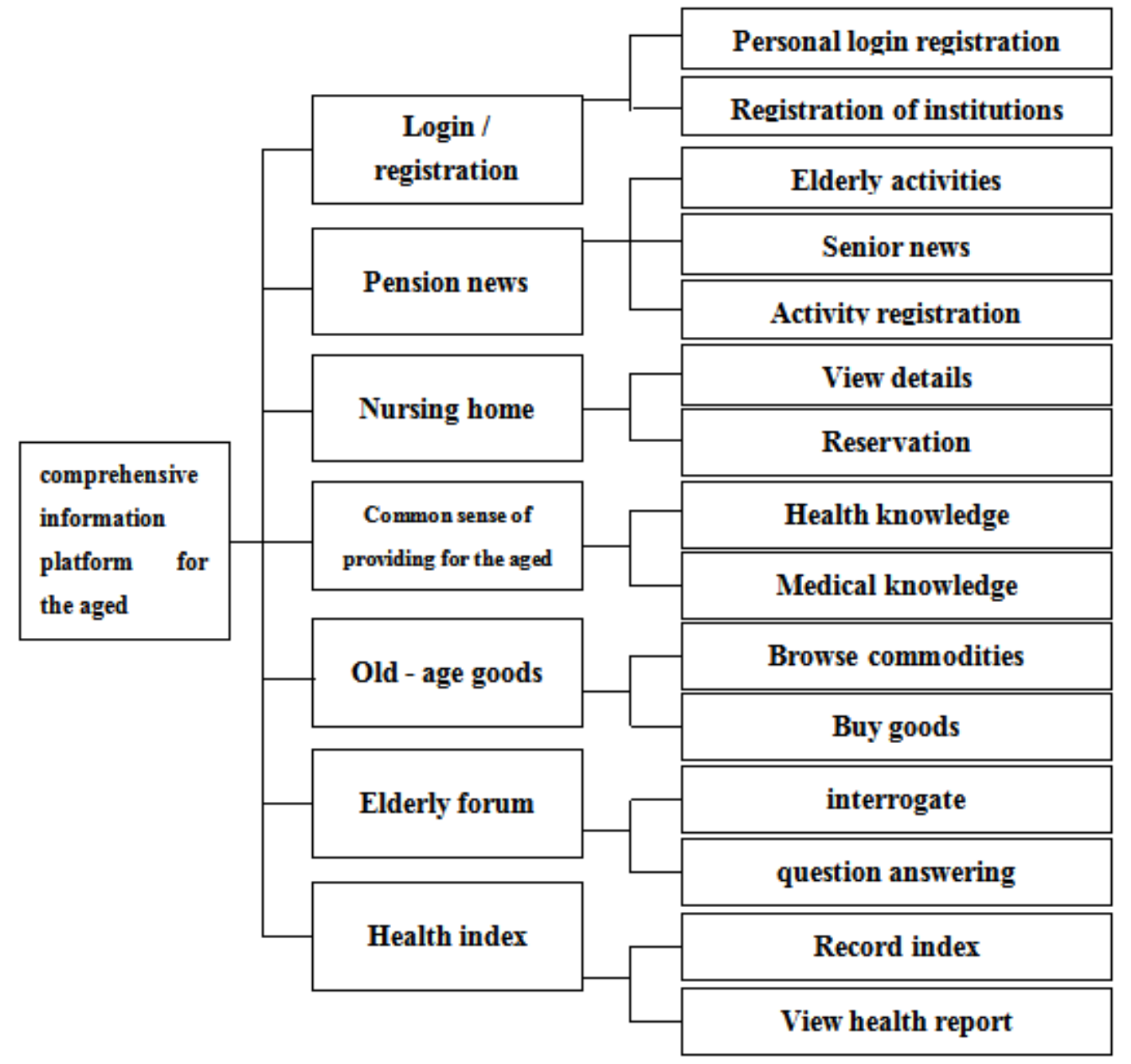

Figure 2. System function module diagram

Login / registration: temporary users can obtain new personal accounts or institutional accounts through registration and $\log$ in. after logging in, they can operate with different user rights respectively.

Pension news: individual users and temporary users can browse endowment dynamics, including recent news and endowment activities, and can participate in the endowment activities of interest. After the number of people is satisfied or the date is approaching, the system administrator confirms the activity and carries out the activity;

Nursing home: users can browse all kinds of nursing homes, including basic information of nursing homes, various kinds of information about rooms they own, various infrastructure facilities they own, optional services, etc. in addition, they can refer to pictures to choose whether to stay in or not.

Common sense of providing for the aged: the system administrator maintains the added common sense. users can browse and understand the basic common sense. 
Old - age goods: users can choose the kind of goods. if they want to buy goods, they can put them into shopping carts to purchase them. after confirmation, they can submit their orders.

Elderly forum: users can express their doubts here, or reply and answer users' questions, and exchange experiences related to endowment.

Health index: the health bracelet worn by the elderly. the index measured in the body fat scale of the home can be uploaded to the platform synchronously by Bluetooth, which is used to record the changes of the physical health index of the elderly, facilitate the analysis of the physical condition of the elderly, and provide reasonable health care suggestions and suggestions.

Administrator module: includes viewing and modifying the information of users and organizations, managing data, such as various order status, system news, pension knowledge, categories and detailed information of pension commodities, and data of elderly materials, etc.

Authority Design. This system mainly includes the function modules of pension news, nursing home, common sense of providing for the aged, old - age goods, elderly forum, health index, etc. it has four levels of user rights, namely browsing users, endowment users, endowment institutions and system administrators.

For temporary users, you can browse the page, view activities, organizations, commodity information, pension knowledge and other information. In addition to the functions of temporary users, the registered elderly users also have the functions of registering login accounts, filling in user information to purchase elderly goods, reserving rooms and services of the elderly care institutions, checking the common knowledge of the elderly, signing up to participate in the elderly care activities, and leaving messages in forums. For the elderly care institutions on the website, they can register and log on official accounts, manage nursing homes, manage room information owned by nursing homes, and manage various services of nursing homes, including medical care, rehabilitation, nutrition and food, domestic service, fitness facilities, etc. The website administrator can not only manage the account information of individuals and organizations, but also manage various order information of users, manage and maintain the content information of pension activities, manage and maintain the content information of pension common sense, maintain and manage the content of news, manage and maintain various information of commodities, and manage the reply information of pension forums.[4,5]

\section{Main Functions and Their Realization}

User recommendation: The system determines the preferences of registered users and nonregistered users by analyzing the information filled in by users, browsing records, and purchased goods and services, and designs different page styles for registered users and non-registered users. When registered users browse different functions of the system platform, they will provide related activities, goods or pension institutions according to their preferences.

Registration function: Firstly, the phone number format is verified by regular expression after the focus is removed from the phone number input box; secondly, after the focus is removed from the repeated password input box, whether the password is the same as the repeated password is verified; finally, the system generates a verification code to judge whether the verification code is consistent; and finally, when clicking the registration button, it verifies whether the nickname is empty. If all the conditions pass, the interface will be called to register, jump to the corresponding servlet, add various information to the user entity, create a new user in the database through the SQL statement in Dao, and add a piece of data.

Realization of check-in function: Users can click on the nursing home to enter the details of the page. after browsing various information and pictures, they can stay in the nursing home. this will jump to the corresponding servlet. first, it will be judged whether the information in the obtained session is empty. if it is empty, a warning bar will pop up to indicate that they are not logged in, and then jump to the login page. If you are logged in, you will jump to the room information and service information details page, where you can select the room you want to stay in and the optional service, submit it with form wrap the required information into entities in servlet, and add SQL statements to the database in Dao. 
Realization of paging function: Since there are many kinds of information in the website, the paging function has become a necessary function. this website uses the paging function in Dao when jumping to the page: by passing the value of the current page and the number of all the information in the database previously obtained to the background as the total number of pages, the corresponding page data is selected from the database when loading the page, written into the Array List and transferred to the foreground, and traversed in JSP with the foreach of the $\mathrm{c}$ tag. At the same time, it can also bring good experience to users. This time the database statement is used for paging. The number of page numbers is passed into the interface as a parameter and then queried.

\section{Conclusion}

Through in-depth and detailed analysis and design of the comprehensive information platform for the aged, the design is based on the MVC structure which is convenient to maintain, has strong expandability and is easy to understand, and uses a variety of layout styles, graphics, animation design, and a user experience good interface. The MySQL database is used to establish a multifunctional background service to ensure the good operation of the website and meet the needs of users. Moreover, the interface is simple, the browsing method is simple, and the website has strong functionality, thus realizing the original design goal.

\section{Acknowledgments}

This thesis is funded by the training program of university student innovative entrepreneurship of Liaoning province.

\section{References}

[1] Y.Y. Ma: Internet of Things Technology. (2018)No.3, p.99. (In Chinese)

[2] H.Y. Hao: Application Research of Web Framework Based on MVC Pattern, Hubei (MS., Wuhan university of technology, China,2013),p.15. (In Chinese)

[3] B. Hu: Design and Implementation of E-Commerce Software Based on Java / Servlet / JDBC Technology, Gansu (MS., Northwestern Technology University, China,2001), p.73. (In Chinese)

[4] Z.J.Gao: Computernik. (2018)No.1,p.11. (In Chinese)

[5] J.Li: Modern Business Trade Industry. (2015)No.7,p.94. (In Chinese) 